\title{
Aeromonas agar is a useful selective medium for isolating aeromonads from faecal samples
}

Aeromonads are still regarded as unusual enteric pathogens. Even though their pathogenic role has not been established (Hanninen et al., 1995), unambiguous convincing evidence suggests that some aeromonads do cause gastroenteritis (Gluskin et al., 1992; Albert et al., 1999). Watery diarrhoea might be accompanied by symptoms such as abdominal pain, fever, and nausea or vomiting; blood in faeces can also appear in serious cases. Aeromonads cause nonresolvable, intermittent diarrhoea that can persist for several months or even years after the initial infection (Janda \& Abbott, 1998). Isolation of aeromonads from human faeces samples is difficult and its success depends on the culture method performed. A valid judgement as to how many aeromonads are involved in diarrhoeal disease is only possible when an appropriate selective medium is used.

Aeromonads, Gram-negative facultatively anaerobic oxidase-positive

glucose-fermenting rods, are ubiquitous waterborne organisms occurring in both fresh and saline waters and in soil.

Aeromonads generally grow on various agars used for screening of enteric pathogens. For their isolation from human faeces, two media have been recommended: cefsulodin-Irgasan-novobiocin agar (CIN), primarily a selective medium for Yersinia enterocolitica, and ampicillin-blood agar (ABA) (Abbott, 2003). ABA has been recommended as a selective medium owing to the beta-haemolytic activity of the majority of clinically relevant Aeromonas species. Because of an increasing ampicillin resistance in other members of the Enterobacteriaceae, it is becoming difficult to screen with oxidase each beta-haemolytic colony that appears. Therefore we decided that ABA is not a suitable medium for the routine laboratory. The next selective medium described for the isolation of Aeromonas spp. is Aeromonas agar (AA; LAB M). AA contains the selective agents brilliant green and Irgasan, which also enable growth of aeromonads susceptible to ampicillin (http://www.lab-m.com/).

In a 2-year survey from 2003 to 2005 , we compared the value of CIN and AA in the isolation of aeromonads. During the first year, routine faeces samples from acute gastroenteritis cases were processed on the following enteric differential media: deoxycholate-citrate agar (DC), MacConkey agar (MC) and CIN. In the second year, the samples were also cultured on AA (Table 1). All media were incubated aerobically at $37^{\circ} \mathrm{C}$ for $18-24 \mathrm{~h}$. Colonies that were typical for aeromonads and that grew on one of the agars mentioned above were cultured on nonselective medium (such as blood agar) and examined for oxidase (OXI-strip; Pliva-Lachema Diagnostika). Isolates were identified as Aeromonas spp. by biochemical kit
ENTEROtest 24 (kit for identification of Gram-negative fermentative rods; Pliva-Lachema Diagnostika); this was confirmed by fatty acid methyl ester analysis using the Microbial Identification System Sherlock (MIDI) (Huys et al., 1994; Čechová et al., 2004).

In our laboratory, faeces samples are inoculated on MC, DC and CIN to screen for common enteric pathogens such as Salmonella spp., Shigella spp., enteropathogenic/enterotoxigenic Escherichia coli or Y. enterocolitica. None of these media are useful for Aeromonas isolation, although aeromonads are able to grow on all of them. On MC or DC the morphology of Aeromonas colonies is similar to that of the non-pathogens which are commonly present in faecal samples and so aeromonads could not be distinguished from them. In addition, the lactose-positive variants of Aeromonas colonies which grew on MC or DC appear falsely

oxidase-negative. On CIN, aeromonads are morphologically indistinguishable from $Y$. enterocolitica or mannitol-positive Citrobacter strains and the oxidase test is usually false negative also. It was demonstrated that the advantage of AA is the possibility of confirming the presence of presumptive Aeromonas by performing an oxidase test directly on a colony grown on the agar. Even the lactose-positive variants of aeromonads, often evaluated as E. coli on

Table 1. Comparison of two various sets of enteric differential media used for screening of aeromonads

\begin{tabular}{|c|c|c|c|c|c|c|c|}
\hline Selective media used & $\begin{array}{c}\text { No. of } \\
\text { faecal } \\
\text { samples }\end{array}$ & $\begin{array}{c}\text { No. of } \\
\text { aeromonads } \\
\text { isolated } \\
(\%)\end{array}$ & \multicolumn{5}{|c|}{ No. of aeromonads isolated with enteric pathogens (\%) } \\
\hline $\mathrm{DC}, \mathrm{MC}, \mathrm{CIN}$ & 20430 & $79(0 \cdot 4 \%)$ & $62(78 \cdot 5 \%)$ & $10^{*}$ & 5 & 0 & 2 \\
\hline
\end{tabular}

${ }^{\star}$ In one sample, Yersinia enterocolitica was also isolated.

tIn one sample, Salmonella sp. was also isolated. 
common enteric differential media, gave a positive oxidase reaction. Moreover, citrobacters appeared on AA as pink colonies and thus were clearly distinguishable from aeromonads. Pseudomonads also grew on AA. They could be differentiated from aeromonads by demonstration of oxidative but not fermentative metabolism (e.g. after inoculating into Hugh and Leifson's O/F medium).

Inclusion of AA in the study more than doubled the frequency of isolation of aeromonads and thus it was considered to be the most efficient selective medium (Table 1). AA is commercially available as a ready-to-use medium that is suitable for routine laboratories. Therefore it was decided to include AA in standard cultivation processing of faeces samples. Compared to other enteric pathogens, Aeromonas spp. were the third most frequent bacteria isolated from patients with acute gastroenteritis (following campylobacters and salmonellas). Aeromonas spp. were isolated in approximately $70 \%$ of diarrhoea cases without confirmation of any other enteric pathogen (Table 1). In these cases we consider that Aeromonas spp. were the cause of the diarrhoea. Faecal specimens were plated on AA directly without enrichment culture, because strains isolated only after enrichment are believed to be not associated with acute diarrhoea (Robinson et al., 1986). We therefore also suppose that all the above-mentioned cases were clinically relevant. Until now, it has appeared that different species and different clones of aeromonads are associated with severe diarrhoea cases (Sinha et al., 2004). Routine use of AA as an additional medium for screening of faecal specimens could elucidate the role of each individual Aeromonas species.

\section{Acknowledgements}

This work was supported by IGA of the Ministry of Health of the Czech Republic, ID code NR/8011-2.

\section{Anna Andělová, Iva Porazilová and Eva Krejči}

Institute of Public Health, Centre for Microbiology, Immunology and Parasitology, Partyzánské nám. 7, 70200 Ostrava, Czech Republic

Correspondence: Eva Krejčí (eva.krejci@zuova.cz)

Abbott, S. L. (2003). Aeromonas. In Manual of Clinical Microbiology, 8th edn, pp. 701-705. Edited by P. R. Murray, E. J. Baron, J. H. Jorgensen, M. A. Pfaller \& R. H. Yolken. Washington, DC: American Society for Microbiology.

Albert, M. J., Faruque, A. S. G., Faruque, S. M., Sack, R. B. \& Mahalanabis, D. (1999). Case-control study of enteropathogens associated with childhood diarrhea in Dhaka, Bangladesh. J Clin Microbiol 37, 3458-3464.
Čechová, L., Durnová, E., Šikutová, S., Halouzka, J. \& Němec, M. (2004).

Characterization of spirochetal isolates from arthropods collected in South Moravia, Czech Republic, using fatty acid methyl esters analysis. J Chromatogr B 808, 249-254.

Gluskin, I., Batash, D., Shoseyov, D., Mor, A., Kazak, R., Azizi, E. \& Boldur, I. (1992). A

15-year study of the role of Aeromonas spp. in gastroenteritis in hospitalised children. J Med Microbiol 37, 315-318.

Hanninen, M. L., Salmi, S., Mattila, L., Taipalinen, R. \& Siitonen, A. (1995). Association of Aeromonas spp. with travellers' diarrhoea in Finland. J Med Microbiol 42, 26-31.

Huys, G., Vancanneyt, M., Coopman, R., Janssen, P., Falsen, E., Altwegg, M. \& Kersters, K. (1994). Cellular fatty acid composition as a chemotaxonomic marker for differentiation of phenospecies and hybridization groups in the genus Aeromonas. Int J Syst Bacteriol 44, 651-658.

Janda, J. M. \& Abbott, S. L. (1998). Evolving concepts regarding the genus Aeromonas: an expanding panorama of species, disease presentations, and unanswered questions. Clin Infect Dis 27, 332-344.

Robinson, J., Beaman, J., Wagener, L. \& Burke, V. (1986). Comparison of direct plating with the use of enrichment culture for isolation of Aeromonas spp. from faeces. J Med Microbiol 22, 315-317.

Sinha, S., Shimada, T., Ramamurthy, T., Bhattacharya, S. K., Yamasaki, S., Takeda, Y. \& Nair, G. B. (2004). Prevalence, serotype distribution, antibiotic susceptibility and genetic profiles of mesophilic Aeromonas species isolated from hospitalized diarrhoeal cases in Kolkata, India. J Med Microbiol 53, 527-534. 